\title{
Implications Organisationnelles Et Humaines De L'introduction Des Tic Pour La Frh : -Cas De L’administration Publique Marocaine-
}

\author{
Meriem Obada (Enseignante-chercheure) \\ Faculté des Sciences Juridiques, Economiques et Sociales -Salé- \\ Université Mohammed V de Rabat, Maroc
}

doi: 10.19044/esj.2017.v13n17p183 URL:http://dx.doi.org/10.19044/esj.2017.v13n17p183

\begin{abstract}
In this study we are interested in the organizational and human implications of ICTs especially for HRF as we see them as more crucial to the effectiveness of the organization as a whole. Mobilizing a contextualist approach, our contribution consists in reconsidering the field of study in order to integrate public administrations, which, like private companies, are strongly challenged by requirements of efficiency and effectiveness. We opted for a quantitative approach by administering a questionnaire to HR managers in about 40 public administrations and used various documents to enrich and complete the field study.

The introduction of ICT in public administration has led to a reorganization of work and a change in working conditions, so the HRF becomes more flexible and communicative. However, interactive services and activities that contribute to skills development are scarce. An ICT culture based on the ethical sense and the mobilization of all the stakeholders is necessary for a complete success of the computerization project of the Public Administration.
\end{abstract}

Keywords: ICT (Information and Communication Technologies), HRF (Human Resources Function), Organizational and Human Implications, Public Administration

\section{Résumé}

Dans cette étude, nous nous intéressons aux implications organisationnelles et humaines des TIC spécialement pour la FRH car nous les considérons comme plus cruciales pour l'efficacité de l'organisation dans son ensemble. Mobilisant une approche contextualiste, notre contribution consiste à reconsidérer le champ de l'étude pour intégrer les administrations publiques, qui à l'instar des entreprises privées, sont vivement interpellées 
par des exigences d'efficacité et d'efficience. Nous avons opté pour une démarche quantitative à travers l'administration d'un questionnaire auprès des responsables $\mathrm{RH}$ d'une quarantaine d'administrations publiques et exploité divers documents pour enrichir et compléter l’étude sur terrain.

L’introduction des TIC dans l’Administration publique a entraîné une réorganisation du travail et une modification des conditions du travail, la FRH devient plus flexible et communicante. Toutefois, les services interactifs et les activités qui concourent au développement des compétences se font rares. Une culture TIC basée sur le sens éthique et la mobilisation de toutes les parties prenantes s’avère nécessaire pour une pleine réussite du projet d'informatisation de l'Administration publique.

Mots clés : TIC (Technologies d'Information et de Communication), FRH (Fonction Ressources Humaines), implications organisationnelles et humaines, Administration Publique.

\section{Introduction}

Les tendances à travers le monde montrent que dans le troisième millénaire les technologies d'information et de communication seront un levier stratégique de tout développement et contribueront de façon importante à l'enrichissement économique des organisations. Dans le domaine de la gestion des ressources humaines, leurs retombées positives sont associées à la mise en œuvre d'un management anticipateur, flexible, qualitatif et personnalisé dont les acteurs sont non seulement la direction des ressources humaines mais toute la hiérarchie voire chaque salarié (Lameul, 2000). Les transformations induites par les TIC affectent les attributions de la fonction ressources humaines en tant que fonction charnière au sein des organisations (Peretti, 1995 ; Guérin et Sire 1999 et Godelier, 1998). Cette fonction qui désigne les responsabilités d'encadrement des personnes (Peretti, 2003) a pour objectif premier de doter toute organisation du personnel nécessaire à son bon fonctionnement. Elle veille aussi bien à l'intégration des ressources humaines (dialogue social et communication de la stratégie) qu’à leur différenciation (recrutement, gestion des carrières, rémunérations individualisées, etc.). Elle représente d'ailleurs, la première fonction organisationnelle qui a profité des TIC pour la gestion de la paie, des effectifs, des congés, du recrutement, de la mobilité, etc. Elle est tout autant considérée comme acteur, support et objet des changements induits par les dites technologies.

Dans ce travail, nous abordons les implications organisationnelles et humaines des TIC spécialement pour la FRH, car nous les considérons comme plus cruciales pour l'efficacité de l'organisation dans son ensemble. Notre contribution consiste à reconsidérer le champ de l'étude pour intégrer 
les administrations publiques dont la gestion est de plus en plus axée sur la qualité, la responsabilité et la performance en vue de fournir le meilleur service aux entreprises et aux citoyens. Au Maroc, les responsables sont aujourd'hui, convaincus que les TIC peuvent être un véritable levier pour la mise à niveau de l'administration publique, appelée à s'adapter et à simplifier ses processus pour réussir le projet de transformation par les TIC. La maîtrise de ces technologies impliquerait nécessairement des changements dans les processus d'affaires, l'organisation du travail, le comportement des fonctionnaires et la culture de gestion des administrations (Megder et al, 2005). La gestion des ressources humaines s'en trouvera pleinement affectée et devra se libérer des activités opérationnelles pour s'occuper davantage des activités stratégiques.

Dans cette étude, notre préoccupation essentielle consiste à répondre au questionnement suivant : « quel est l'impact de l'introduction des TIC dans l'administration publique sur la FRH des points de vue organisationnel et humain ?». Pour ce faire, nous avons mobilisé une approche contextualiste et opté pour une démarche quantitative à travers l'administration d'un questionnaire auprès des responsables RH d'une quarantaine d'administrations publiques et exploité divers documents pour enrichir et compléter l'étude sur terrain.

Pour notre plan de travail, nous allons nous intéresser d'abord, d'un point de vue théorique, aux différentes mutations que connait la FRH avec l'avènement des TIC pour ensuite délimiter les contours de notre travail de terrain qui se veut une contribution à l'étude de l'impact de ces technologies sur la gestion publique particulièrement celle liée aux ressources humaines et aux problématiques qu'elle ne manque de susciter.

\section{L'évolution de la FRH sous l'impulsion des TIC}

Les TIC désignent la combinaison de l'équipement, du matériel, des procédures et des systèmes utilisés pour recueillir, emmagasiner, analyser et diffuser l'information afin qu'elle puisse se traduire en savoir (DeSanctis, 1995). Les TIC, le Web et l'ordinateur sont trois éléments quasiindissociables qui ont profondément transformé la conception organisationnelle en permettant d'acquérir de nouvelles compétences. Avec leur avènement, la FRH, comme sous-système organisationnel, a connu de profondes mutations au niveau de son fonctionnement, ses processus et ses missions (Kalika, 2002). 


\section{D'une FRH administrative à une FRH stratégique \\ Les TIC se substituent à certaines activités d'exploitation ainsi qu'à certains mécanismes de contrôle}

Dans un grand nombre d'organisations où la bureaucratie mécaniste s'imposait particulièrement dans le secteur public, l'apparition des technologies d'information, qui n'étaient pas encore intégrées aux technologies de communication, a modifié les postes de travail les plus routiniers, les plus spécialisés et les plus répétitifs (Woiceshyn, 1997; Schilling, 1998). Par la suite, des systèmes informatisés d’aide à la décision ont pu remplacer certains manuels de politiques, procédures et directives ainsi que les multiples mécanismes de contrôle des processus associés à ce type de structure organisationnelle (Osborne et al, 1984). Les solutions électroniques mises en place ont en commun de dématérialiser et d'optimiser les processus RH (Nahon et Taskin, 2009). Brilman et Hérard (2006) soulignent que la généralisation des architectures informatiques en réseau a un impact majeur sur la circulation transversale de l'information, ce qui entraîne, dans la plupart des cas, la réduction du nombre de niveaux hiérarchiques. Les Intranet et Internet abolissent les frontières entre départements, contribuant ainsi à mailler horizontalement tous les collaborateurs d'une organisation. La productivité augmenterait sous l'effet de l'utilisation des ordinateurs ce qui tendrait à indiquer que l'utilisation de l'ordinateur et l'introduction de pratiques en matière de ressources humaines vont de pair (OCDE, 2003). A cet égard, le rôle des SIRH $^{45}$ (systèmes d'information des ressources humaines) est décisif puisqu'il permet non seulement de fournir des informations pertinentes aux acteurs locaux mais aussi de collecter des données pour les agréger et les utiliser à un niveau décisionnel plus élevé (Niederman, 1999). L’objectif est de rationaliser, automatiser et éliminer les fonctions répétitives et routinières et si possible, contribuer à renforcer les responsabilités « stratégiques » du professionnel en ressources humaines en tant que partenaire stratégique d'affaires et agent de changement ${ }^{46}$.

\footnotetext{
45 Tannenbaum (1990) définit le SIRH comme étant : « Un système utilisé pour acquérir, stocker, manipuler, analyser, trier, distribuer des informations pertinentes concernant les ressources humaines dans une organisation ». Toutefois, Hendrickson (2003) précise qu’il n’est pas restreint à la technique (matériels informatiques et applications logicielles) mais intègre aussi les individus, les procédures et politiques ainsi que les données requises pour gérer la fonction $\mathrm{RH}$.

${ }^{46}$ Par opposition à deux rôles plus traditionnels et à caractère opérationnel : celui de représentant de personnel et d'expert de processus administratifs.
} 


\section{Les TIC représentent un potentiel stratégique}

Ruel (2005) affirme qu'il faut différencier le SIRH et l'e-RH, car le SIRH est destiné à la fonction RH alors que l'e-RH concerne les acteurs extérieurs au département RH. Deux objectifs sous-tendent la mise en place de l'e-RH (Nahon et Taskin, 2009) :

- faciliter l'accès aux données et aux ressources, ce qui facilite et accélère la prise de décision pour les gestionnaires et les professionnels $\mathrm{RH}$; et

- $\quad$ procurer aux employés un accès personnalisé à de nombreuses applications offertes par le service des ressources humaines.

Dans le même sens, Silva (2001) et Peretti (2001) considèrent que l'utilisation des TIC modifie les modes de gestion de la fonction RH au point que l'on évoque la génération dans le futur d'une e-DRH. Trois types d'évolution sont à distinguer:

- $\quad$ le libre-service ressources humaines (LSRH) qui s'inscrit dans un mouvement général de suppression des emplois administratifs peu qualifiés remplaçables par l'ordinateur. Il confie au salarié certains aspects de sa propre gestion (gestion du temps et des congés, inscription en formation, bilan de compétences, mise à jour du dossier individuel, etc.) ;

- la modification du pouvoir hiérarchique dans la mesure où le manager opérationnel devient le « premier $\mathrm{RH}$ »; et

- l'externalisation des processus RH telles que la gestion de la paie ${ }^{47}$ et la formation. Celle-ci est confiée à des prestataires de services externes, et pourrait même être disponible sous la forme de e-Learning conçue par des entreprises de formation.

Allègre et Andréassian (2008) considèrent que tous les processus RH utilisant des logiciels sont susceptibles, à plus ou moins longue échéance, d'être traités en libre service par le salarié ou le cadre hiérarchique ou en partie être externalisés. Les experts de la GRH (moins nombreux mais de plus haut niveau) peuvent se consacrer à des missions dites stratégiques, c'est-à-dire directement liées au développement de l'entreprise et de sa compétitivité (Picq, 2000 ; Ulrich, 2001).

Les TIC peuvent être source de compétences diverses et améliorer l'efficacité, la rapidité d'adaptation et le rendement des activités d'exploitation (Lei et al, 1996 ; Palvia et Roche, 1996). Elles donnent aux travailleurs de tous les paliers hiérarchiques l'information dont ils ont besoin pour planifier leurs actions, les coordonner à celles des autres et contrôler leurs propres activités.

${ }^{47}$ La gestion de la paie se prête particulièrement à la sous-traitance par son caractère régulier (saisie de données), cyclique (échéances mensuelles, annuelles), par sa nécessaire ponctualité et par la complexité des rémunérations. 


\section{Changement organisationnel et comportement des acteurs}

L'impact des TIC n'affecte pas uniquement la dimension organisationnelle du travail mais il peut aussi concerner les conditions et le climat du travail et influencer les attitudes et comportements des collaborateurs face au changement. La négligence du facteur humain peut être à l'origine de situations conflictuelles et donc de nombreux échecs de projets informatiques.

\section{Climat du travail}

La motivation et l'implication des salariés augmenteraient sous l'impulsion de l'informatisation des systèmes RH perçue comme un signe d'amélioration des conditions de travail et de stabilité de l'emploi (Matmati, 2002). Les TIC stimuleraient l'autonomisation des salariés et donneraient plus de portée à leur poste de travail, le rendant plus intéressant et sollicitant une plus grande implication dans la reconnaissance et la résolution de problèmes (Markus et al, 2002 ; Beyer et al, 1997). Le salarié d'exécution prendrait en charge la gestion de ses affaires courantes et son propre développement professionnel. Il évoluerait entre responsabilité et autonomie et deviendrait le premier client à satisfaire (Matmati, 2002). Selon la plupart des nouvelles théories managériales, les TIC faciliteraient la communication entre les individus, entre les différents services comme entre les entreprises et favoriseraient le passage à une organisation moins centralisée. La GRH devient plus performante, plus efficace et plus intelligente (Guérin et Wils, 2002) surtout dans un contexte de gestion intégrale de la qualité qui se base sur le travail d'équipe et l'habilitation du personnel ; facteurs clés de réussite des organisations contemporaines (Veiga et Dechant, 1997).

Toutefois, d'après certaines études l'impact des TIC sur l'autonomisation et la qualification des salariés est plutôt mitigé (Moatty et Gollac, 2001 $)^{48}$. Bien qu'il soit synonyme dans certains cas d'une forte décentralisation des décisions et d'un accroissement des circulations horizontales de l'information, il n'implique pas une diminution du contrôle. Dans le secteur public, si une certaine forme de contrôle disparait, l'évaluation des agents est renforcée, en s'appuyant directement sur les possibilités d'enregistrement et de mobilisation des informations que permet l'informatique (Duvernay et Marchal, 1994). De plus, si l'informatique s'implante dans une organisation moins centralisée, elle se traduit par d'importants conflits organisationnels et une augmentation du contrôle sur les travailleurs de base, avec la mesure en temps réel de leur productivité individuelle (Moatty et Gollac, 2001).

\footnotetext{
${ }^{48}$ Cité dans Gollac, M. (2003), «Les métiers face aux technologies de l’information », in [http://www.ladocumentationfrancaise.fr/var/storage/rapports-publics/034000182.pdf], consulté le 26/11/2016.
} 
Les TIC impliquent un mode d'organisation en réseau qui exige de chacun un engagement individuel et personnel de plus en plus fort et génère une surcharge quantitative et qualitative du travail au détriment des loisirs et de la vie privée. Le salarié doit s'approprier de nouveaux outils de travail et réaliser dans de nombreux cas de nouvelles tâches, souvent complexes, nécessitant un apprentissage continu et une coopération accrue ${ }^{49}$ (Kalika et al., 2005 ; Gollac , Greenan et Hamon, 2000). Stress, fatigue mentale et cognitive apparaissent sans supprimer la fatigue physique plus spécifique à la société industrielle (Lasfargue, 2003). L'usage des TIC implique aussi une dépendance accrue des utilisateurs du bon fonctionnement des outils informatiques, des cloisonnements importants des échanges et des collectifs de travail car les salariés auraient moins tendance à discuter avec leurs collègues ce qui réduit leur sociabilité et les échanges directs (Lachmann et al, 2010).

\section{Résistance au changement}

L'introduction des TIC n'est pas vécue de la même manière par tous les membres de l'organisation qui peuvent développer une forte résistance au changement. En effet, certains salariés y voient une source d'exclusion sociale du fait que la maitrise de chaque nouvelle génération de technologie dépend très fortement du capital culturel personnel, du niveau des responsabilités hiérarchiques et de l'âge du salarié (Gollac, 2003) ${ }^{50}$. Les TIC exigent plus de réactivité de la part des collaborateurs, or certains ont du mal à se représenter la réalité au delà des données affichées sur l'écran (Lasfargue, 2000). Ce blocage ne dépend pas de leur niveau d'études mais peut être dû à un manque de confiance en soi et en ses compétences ${ }^{51}$ (Bailly et $\mathrm{al}, 2009$ ) ou encore à des peurs irrationnelles ${ }^{52}$ (Dukan, 2003). De plus, les individus ont le sentiment d'être surveillés du fait que les TIC permettent de numériser, observer et stocker toutes les actions réalisées sur les réseaux de communication.

En relation avec les développements théoriques, il apparait clairement que l'introduction des TIC dans les organisations bouleverse les méthodes de travail, les paradigmes dominants et les attitudes. Il s'agit donc

\footnotetext{
${ }^{49}$ La mise en œuvre de cette coopération demande une implication personnelle plus forte qui se traduit par une pression accrue des délais, des dépassements d’horaires plus fréquents ce qui nécessite de repenser le système d'évaluation et de rétribution.

50 La Documentation française (2003), « Les métiers face aux technologies de l'information », in [http://www.ladocumentationfrancaise.fr/var/storage/rapportspublics/034000182.pdf], consulté le 26/11/2016.

${ }^{51}$ Les TIC exigent la gestion d'une forme de travail abstraite, d'une information abondante et de temps nécessaire à l'apprentissage, pour l'exploitation ou le dépannage.

52 Forte confusion, perte de contrôle, refus d'apprendre et de se mettre à jour continuellement, etc.
} 
d’un véritable changement dans les processus managériaux en particulier ceux liés à la FRH. Se pose maintenant la question de savoir, quelle est la portée et l'impact de ce changement dans le contexte des administrations publiques au Maroc?

\section{Protocole et résultats de la recherche}

\section{Contexte de la recherche}

Aujourd'hui, l'Etat est placé devant les mêmes exigences d'efficacité et d'efficience subites par l'entreprise. Il est confronté à des contraintes financières très sévères, à une compétition internationale de plus en plus intense, ainsi qu'aux exigences toujours plus grandes des usagers. La modernisation de l'Administration publique marocaine devient de plus en plus un défi incontournable à relever surtout avec la révolution silencieuse imposée par les progrès de l’informatique et des télécommunications. Le gouvernement électronique, l'e-RH, les télé-services, l'Intranet, etc. ; font maintenant partie des priorités des hauts dirigeants de l'Etat pour une meilleure valeur ajoutée publique.

En 2013, l'Administration marocaine disposait de 466 sites Web ${ }^{53}$ (148 pour les départements ministériels, 103 pour les grands établissements publics et 215 pour les autres établissements publics), c'est-à-dire que 86.84\% des départements ministériels disposent d'un Portail Internet contre $63.60 \%$ pour les établissements. Le taux d'équipement en ordinateurs (nombre de postes rapporté à l'effectif) était de 31,22\% en 2009. Les départements ministériels et les grands établissements publics (offices, grandes entreprises, etc.) dépassaient cependant les $47 \%$ alors que les autres établissements publics n’étaient équipés qu’à hauteur de 17,24\%.

Toutefois, ce n’est pas le taux d’équipement en matériel informatique en soi qui est garant de l'efficacité numérique d'une Administration mais plutôt l'usage qu'elle en fait et qui se manifeste pleinement dans le concept de «nouvelle gestion publique ». Celle-ci n’est pas sans entraîner des changements dans les processus d'affaires, les structures d'emplois, l'organisation du travail, le comportement des acteurs et la culture même de gestion des Administrations publiques. Une gestion stratégique des ressources humaines est donc nécessaire pour accompagner le rythme accéléré des innovations technologiques et améliorer la productivité.

Cette nouvelle stratégie s'inscrit dans la mouvance de ce qu'on appelle le Nouveau Management Public (NMP) qui depuis les années 80 appelle les gouvernements à rechercher une plus grande efficience de

53 Ministère de la fonction publique et la modernisation de l'Administration, 2013, Cartographie de l'utilisation des Technologies de l'Information et de la Communication (TIC) dans les secteurs publics, in [http://www.mmsp.gov.ma/ carto2013/] consulté le 20/11/2016. 
l'action publique à travers une modernisation des administrations et des services publics qui opère de profondes recompositions dans les systèmes d'actions publics locaux et nationaux. En effet, le NMP a inspiré les réformes de l'État, d'abord dans les pays anglo-saxons (Grande Bretagne, Nouvelle-Zélande et Etats Unis) à la fin des années 1970 pour essaimer un peu partout durant les décennies suivantes, dans la plupart des pays de l'OCDE notamment. Quelques principes se dégagent néanmoins (Boston, 2010): 1/ le secteur public peut et doit être managé selon les mêmes règles que le secteur privé ; l'esprit et les valeurs de l'action publique changent ; 2/ l'accent est plus mis sur le résultat de l'action publique que le respect des procédures ; des objectifs quantitatifs et des mesures chiffrées de l'efficience sont mis en place et $\mathbf{3}$ / la relation hiérarchique est également transformée, le principe étant de privilégier l'autonomie des agents, en contrepartie d'un renforcement des mécanismes de contrôles (suivi d'indicateurs, rapports d'activité, etc.). Toutefois, si le NMP correspond à une volonté de modernisation de l'appareil de gestion publique, il ne peut en aucun cas porter atteinte aux principes fondamentaux des services publics (Pettigrew, 1997). Il ne se résume pas non plus à la substitution d'un modèle privé à un modèle public mais plutôt à l'élaboration progressive d'un nouveau modèle hybride, non encore achevé (Alecian et Foucher, 2002).

A ce titre, l'une des principales valeurs et formes de rationalité que prône ce paradigme managérial est la culture de la performance dans les organisations publiques. Or, il est largement reconnu aujourd'hui que la gestion des ressources humaines contribue à cette performance et que l'impact des TIC sur la FRH ne permettrait que de consolider l'efficacité et l'efficience de l'action publique. En lien avec ces nouvelles technologiques, un renouveau stratégique de la GRH est rendu possible pour marquer le passage à un management humain plus créateur de valeur et qui s'émancipe d'une mission traditionnellement administrative ${ }^{54}$ réductrice et obsolète. L'objectif est de réorganiser la FRH par: 1/ la reconfiguration de ses processus opérationnels et structures dans le sens d'une meilleure adaptation des profils aux postes de travail ; 2 / son repositionnement dans le dispositif de pilotage de l'entreprise par l'entremise d'un système d'information intégré permettant de prendre les meilleures décisions en matière d'emploi et de GRH publics. En somme, les TIC favoriseront «l'émergence d'un nouveau modèle de GRH caractérisé par (Matmati, 2000) :

- $\quad$ un libre accès à l'information RH au profit aussi bien des managers que des salariés ;

\footnotetext{
${ }^{54}$ Qui se limite à la gestion des dossiers administratifs du personnel en matière notamment de recrutement, mobilité, rémunération, etc.
} 
- $\quad$ une forte réduction des tâches administratives à charge de la FRH du fait des «prestations en ligne » que l'intranet apportera tant aux managers qu’aux salariés ;

- la proposition de «prestations de GRH en ligne » touchant des domaines comme le recrutement, la formation, l’évaluation des performances et la mobilité interne ;

- $\quad$ une responsabilisation totale des managers opérationnels sur la GRH de leurs structures respectives ;

- $\quad$ une FRH à l'écoute des managers pour identifier leurs besoins et proposer les solutions appropriées ; et

- une FRH orientée sur des missions stratégiques d'appui aux managers, de conduite de changement, de développement de l’apprentissage organisationnel ».

C’est pourquoi, notre recherche poursuit un double objectif:

- $\quad$ identifier et analyser les différentes mutations de la FRH en relation avec l'introduction des TIC dans l'administration publique et ce à deux niveaux: organisationnel et humain à la lumière des développements théoriques en la matière ; et

- formuler des propositions pour une meilleure optimisation de l'impact des TIC sur la modernisation de l'Administration publique par le biais d'une bonne gestion des ressources humaines.

\section{Méthodologie}

Le choix de l'approche contextualiste ${ }^{55}$ se justifie par notre volonté de saisir l'impact de l'introduction des TIC sur la FRH dans l'administration publique au Maroc et ce de deux points de vue: organisationnel (réorganisation du travail) et humain (perceptions et comportements des acteurs). Le choix de cette méthode nous a conduits à mobiliser d'une part une démarche quantitative à travers l'administration d'un questionnaire auprès des responsables RH ou de gestion de personnel d'une quarantaine d'administrations publiques. Nous avons également exploité divers documents, pour l'essentiel produits par le ministère de la fonction publique et de la modernisation de l'Administration publique, pour enrichir et compléter l’étude sur terrain.

La description de notre échantillon est résumée dans le tableau 1 ciaprès :

\footnotetext{
${ }^{55}$ Le contextualisme a surtout été développé par Pettigrew (1985) qui se pose la question de comprendre « comment et pourquoi les changements dans la fonction ressources humaines se produisent dans différents contextes sous différentes conditions socio-économiques, à travers le temps ». Cornet (1995) souligne que le contextualisme est basé sur la mise en relation du contenu de changement et de son contexte, autant interne qu'externe, dans une dimension processuelle et nécessairement temporaire.
} 
Tableau1 : Présentation de l'échantillon

Administrations

Les Départements Ministériels

Les Grands Etablissements Publics

Effectif

$15 \quad 38$

Les Autres Etablissements Publics

Total

$14 \quad 35$

$11 \quad 27$

40

100

Source : élaboré par l’auteur.

Nous avons réparti notre échantillon en trois types d'entités administratives :

- $\quad$ Les Départements Ministériels (DM).

- $\quad$ Les Grands Etablissements Publics (EP1) qui ont généralement une envergure nationale et disposent, ou sont censés disposer, d'un système d'information avec un niveau élevé d'utilisation des nouvelles technologies.

- Les autres Etablissements Publics (EP2) qui comprennent des établissements publics agissant essentiellement au niveau régional ou local.

Ce choix rend compte de la grande hétérogénéité des organismes publics dont certains sont équipés de technologies de pointe alors que d'autres ne disposent que d'une infrastructure rudimentaire dédiée essentiellement aux travaux bureautiques.

Pour l'enquête terrain, nous avons mobilisé tantôt la messagerie électronique, tantôt le contact direct et nous avons pu obtenir 30 questionnaires renseignés soit un taux de réponse de $75 \%$ (soit 12 DM, 10 EP1 et 8 EP2) et que nous jugions très satisfaisant vu la grande difficulté d'accès à l'information.

Le questionnaire a été construit autour de plusieurs préoccupations :

- $\quad$ L’usage des TIC.

- $\quad$ Le contenu des TIC.

- $\quad$ L’impact des TIC sur la gestion des ressources humaines.

- $\quad$ L’impact des TIC sur la restructuration de la FRH.

- $\quad$ L’impact des TIC sur le climat du travail.

- $\quad$ Les TIC et les problèmes opérationnels.

La réponse à ces préoccupations permet de juger de l'impact théorique présumé positif des TIC sur la performance des organisations en insistant sur le rôle de premier plan de la FRH dans l'appropriation de ces technologies. De la même façon, les TIC sont censées aider les services RH à anticiper et mettre en œuvre les changements nécessaires à la structuration et au bon fonctionnement de cette fonction clé. L’introduction des TIC se répercuterait également sur le climat du travail en interpellant vivement tout le personnel sur ses nouveaux rôles en milieu de travail. 


\section{Résultats et perspectives de l'étude}

Pour les besoins de notre recherche, les résultats ont été synthétisés par thème pour capturer les implications organisationnelles et humaines du renouveau technologique au sein de l'administration publique au Maroc du point de vue des responsables ressources humaines ou de gestion du personnel.

\section{Usage et contenu des TIC}

Toutes les entités enquêtées déclarent disposer d'une structure informatique ce qui dénote de l'importance accordée à l'informatique par l'administration publique en général. En moyenne, le personnel utilisateur d'ordinateurs représente environ $47 \%$ de l'effectif total et dans $80 \%$ des administrations (au nombre de 24), le taux d'utilisation d'ordinateurs est supérieur à $50 \%$.

Pour 97\% des administrations (29 entités), l’Internet demeure l'outil le plus utilisé pour les besoins du travail, suivi de la messagerie électronique avec un taux de 90\% (27 entités), puis l'Intranet avec un taux de 80\% (24 entités), l'ERP ${ }^{56}$ avec 24\% (7 entités) comme l'illustre le graphique 1 cidessous. Une seule administration utilise le Workflow ${ }^{57}$ alors que le Groupeware $^{58}$ n'est utilisé par aucune administration.

GRAPHIQUE 1 : Classement des TIC par nature et fréquence d'usage

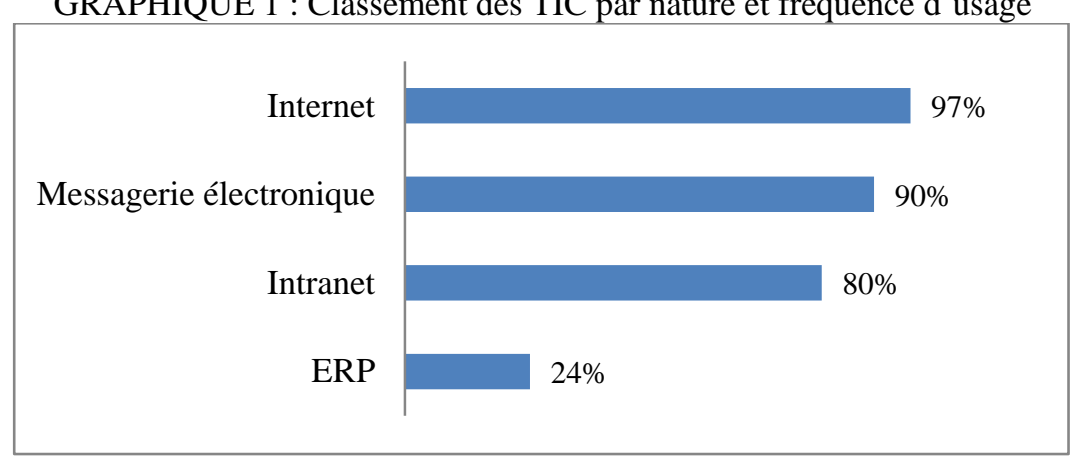

${ }^{56}$ Les ERP (Entreprise Resource Planning) ou Progiciels de Gestion Intégrée sont des applications dont le but est de coordonner l'ensemble des activités d'une entreprise (activités dites verticales telles que la production, l'approvisionnement ou bien horizontales comme le Marketing, la force de vente, la GRH, etc.) autour d'un même système d'information.

${ }^{57}$ Le WorkFlow ou Gestion électronique des processus métier représente les interactions sous forme d'échange d'informations entre divers acteurs tels que des humains, des applications ou services et des processus tiers, etc. il fournit à chacun des acteurs, les informations nécessaires pour la réalisation de sa tâche.

${ }^{58}$ Le Groupeware ou logiciel de groupe permet à un groupe de personnes de partager des documents à distance pour favoriser le travail collaboratif. 


\section{TIC et gestion des ressources humaines}

Toutes les administrations enquêtées déclarent que les TIC sont mobilisées au service de la gestion administrative des dossiers du personnel. $43 \%$ les utilisent pour la gestion de la mobilité, $40 \%$ pour l'e-recrutement, $37 \%$ pour la gestion des connaissances, $33 \%$ pour les tableaux de bord sociaux, 30\% pour le libre-service RH, $23 \%$ pour l'e-Learning et $17 \%$ pour d'autres services : gestion électronique des documents, pointage, promotion, etc.

GRAPHIQUE 2 : Fréquence des AP qui utilisent les TIC au service de la GRH

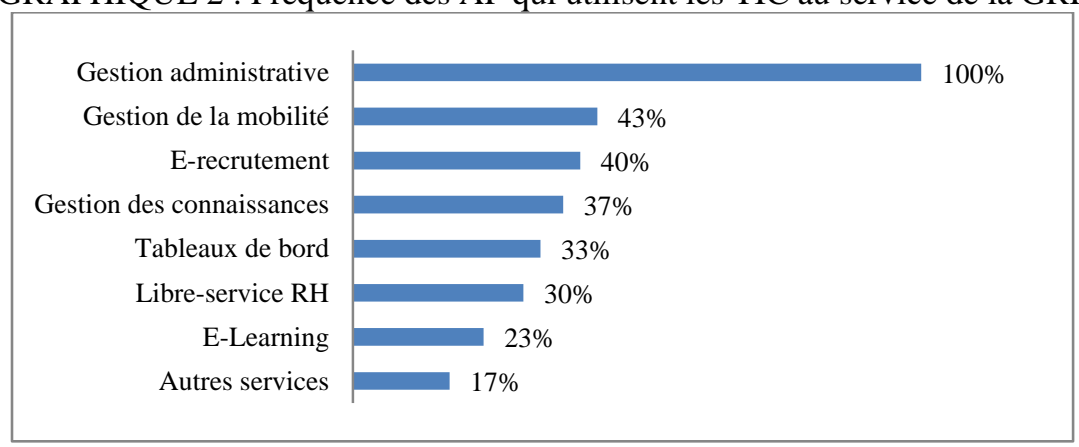

L'utilisation des TIC se fait donc essentiellement au profit de la gestion administrative des effectifs (gestion du personnel et des informations sociales). Toutefois, l'e-RH implique une gestion plus dynamique des personnels dans la mesure où l'administration publique a opté, bien que partiellement, pour une décentralisation de l'information et une plus grande responsabilisation de ses ressources humaines. Gestionnaires et salariés sont désormais impliqués dans la réalisation de tâches qui étaient auparavant exclusivement dévolus aux spécialistes des ressources humaines (erecrutement, e-Learning, e-évaluation, libre-service RH) (Kalika et al, 2005). La DRH pourra ainsi se concentrer sur des activités plus stratégiques et créatrices de valeur telles que la gestion des connaissances, la gestion des carrières, etc. (Peretti, 2001 ; Silva, 2001 et Picq, 2000).

D’ailleurs, les TIC ont contribué à une amélioration de la productivité dans $90 \%$ des administrations objet de l'enquête (soit 27 entités) grâce notamment à la rationalisation des coûts de fonctionnement et à l'amélioration de la qualité du traitement des dossiers. Seulement 3 administrations déclarent que l'usage des TIC n'a pas influencé le niveau de productivité.

En matière de communication interne et comme l'illustre le graphique 3, plus de la moitié des administrations enquêtées considère qu'avec les TIC, l'échange d'informations s'est considérablement amélioré entre les différents paliers hiérarchiques et seulement une minorité le considère rare et attribue ce manque au style de management exercé par le supérieur hiérarchique. 
GRAPHIQUE 3 : Importance de l'échange d'informations suite à l'introduction des TIC

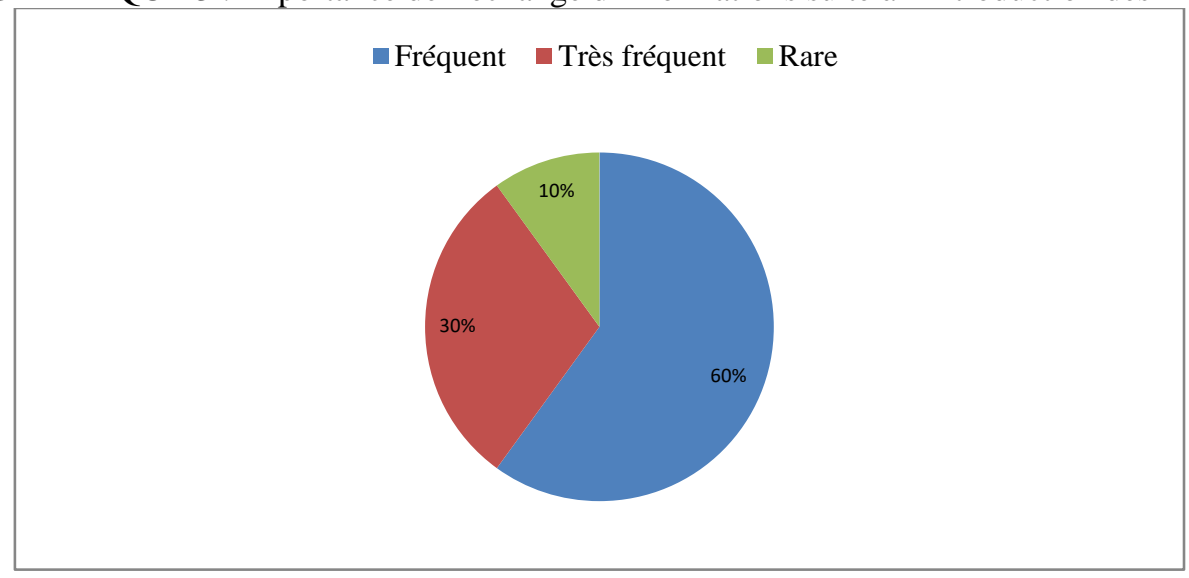

Pour démocratiser l'accès à l'information, son management devient prioritaire surtout pour transformer les connaissances disponibles dans l'administration en savoir-faire organisés (Lei et al, 1996 ; Palvia et Roche, 1996). Dans ce sens, 90\% des administrations enquêtées ont constaté que les TIC ont contribué à l'amélioration des connaissances du personnel surtout celles dédiées aux métiers en lien direct avec ces technologies et aux métiers de la GRH (acquisition de nouvelles compétences).

\section{TIC et restructuration de la FRH}

$77 \%$ des administrations enquêtées ont procédé à la réorganisation du travail suite à l'introduction des TIC (7 \% l'ont effectué plusieurs fois). Pour la majorité d'entre elles (70\%), cette réorganisation était satisfaisante car elle a impliqué une rationalisation des tâches pour une meilleure efficacité et responsabilisation des collaborateurs. Pour les $17 \%$ qui ont conservé leur ancien mode d'organisation, elles l'expliquent par le souci de respecter les normes et procédures du travail à la faveur d'une centralisation du pouvoir de décision caractéristique des bureaucraties mécanistes. D’ailleurs, toutes les administrations déclarent que l'introduction des TIC a permis de diminuer certains postes sans se traduire pour autant par leur suppression ce qui nous amène à penser que les TIC n'ont pas eu d'impact sur la déhiérarchisation de la FRH. Toutefois, $80 \%$ des administrations enquêtées ont constaté que le travail en groupe est de plus en plus fréquent ce qui renforce la collaboration et la cohésion du personnel.

Les TIC ont aussi supporté l'implantation du SIRH par la majorité des administrations étudiées (70\%) ; ce qui a simplifié et facilité l'exécution des activités administratives. Bien plus, ce système a contribué à extraire des informations essentielles et a écourté les délais pour la prise de décision des 
acteurs impliqués dans la GRH; responsables RH et cadres notamment (Nahon et Taskin, 2009).

\section{TIC et climat du travail}

\section{- $\quad$ Conditions de travail}

La majorité des administrations soit 90\% (27 administrations) ont constaté que les TIC ont augmenté le rythme du travail ce qui a été à l'origine d'un surcroît de stress chez les employés de 8 administrations (27\%). A l'inverse, 6 administrations (20\%) déclarent que le niveau de stress a diminué grâce à l'usage des TIC. D'ailleurs, pour 77\% des administrations enquêtées (au nombre de 23), l'introduction des TIC n'a pas créé un sentiment d'isolement chez les utilisateurs et seulement 13\% (4 entités) déclarent que les conflits entre la hiérarchie et les subordonnés augmentent sous l'influence des TIC.

Ainsi, 20 administrations (67\%) ont déclaré qu'elles ont renforcé le contrôle du personnel dans l'exécution de son travail. De plus, 18 administrations (60\%) ont constaté que la motivation et l'implication du personnel se sont ameliorées en raison du développement de leur autonomie (pour $83 \%$ des administrations), à l'amélioration de la qualité du travail et dans une moindre mesure à l'enrichissement des tâches. Ces différents constats sont synthétisés dans le graphique 4 ci-après :

GRAPHIQUE 4 : Fréquence des AP ayant connu une modification des conditions de travail

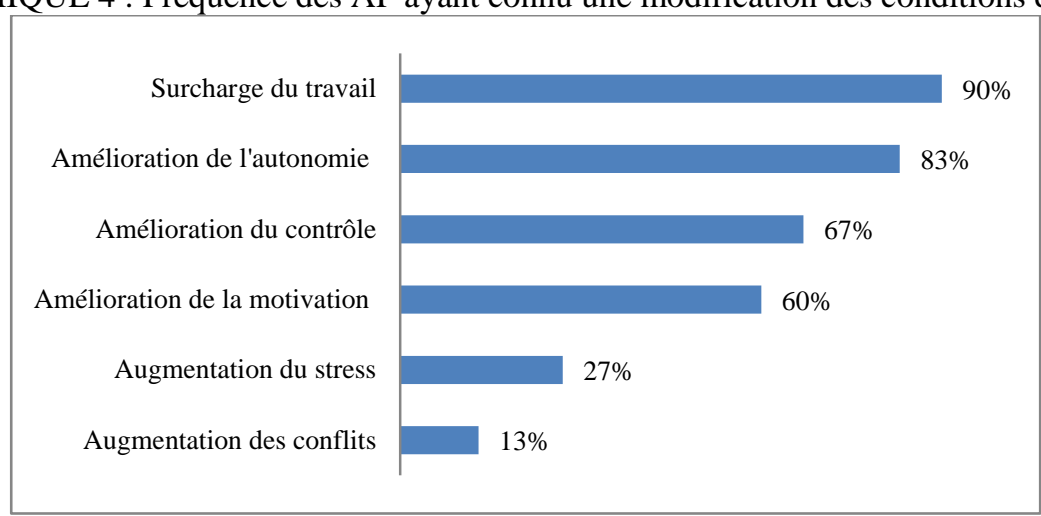

\section{- $\quad$ Développement des compétences}

Comme l'illustre le Graphique 5 ci-dessous, seulement 34\% des administrations enquêtées (soit 10 entités) ont constaté que l'usage des TIC a permis l'acquisition de nouveaux savoirs et savoir-faire chez les utilisateurs : les TIC ont amelioré la réactivité du personnel (dans 22\% des entités), l'innovation (dans 19\% des entités), l'initiative (dans 15\% des entités) et $11 \%$ ont déclaré que les TIC ont permis de développer la capacité d'anticipation de leurs salariés. 
GRAPHIQUE 5 : Fréquence des AP ayant assisté à un développement des compétences

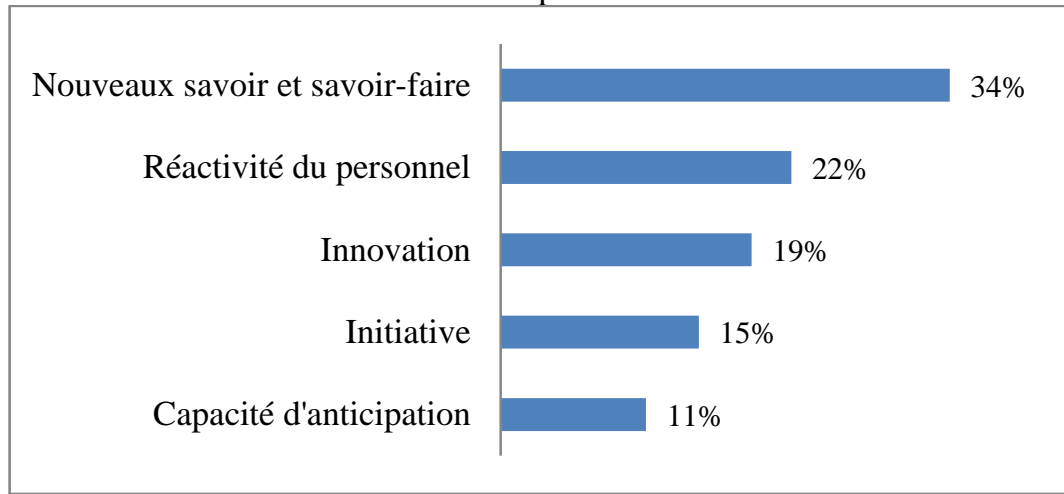

\section{- $\quad$ Résistance au changement}

20 administrations (67\%) ont enregistré peu de cas de résistance au changement dus à l'introduction des TIC, 5 administrations (17\%) ont enregistré beaucoup de résistance et 4 administrations (13\%) n’ont pas enregistré de cas de résistance comme indiqué dans le graphique 6 ciaprès :

GRAPHIQUE 6 : Effectifs et Fréquences des AP ayant enregistré une résistance au changement

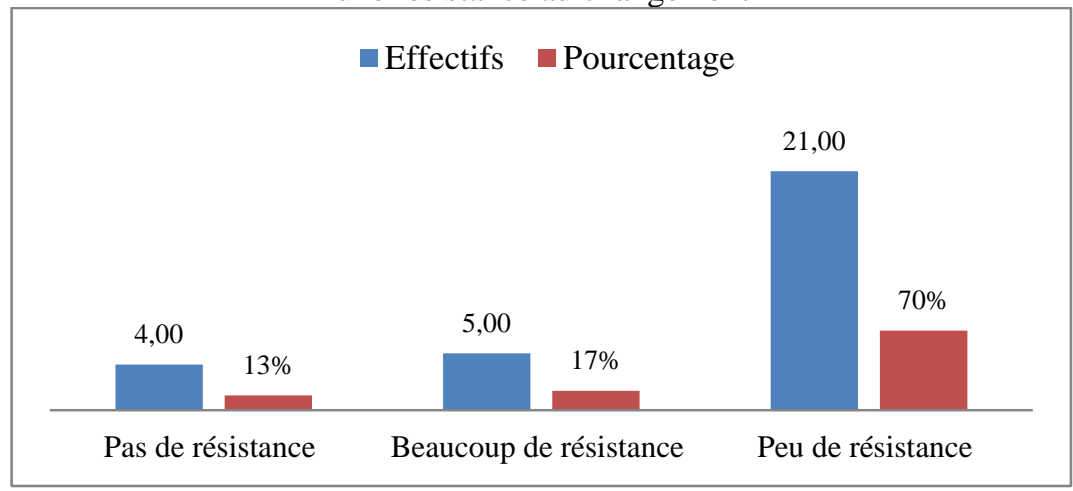

Le refus de s'occuper de nouveaux dossiers constitue le principal comportement de résistance (11 administrations), suivi par le refus d'utilisation des TIC (8 administrations) enfin, le refus de suivre une formation en TIC (4 administrations).

Les causes de résistance repérées sont multiples: l'âge des utilisateurs en constitue la principale cause avec 60\% des réponses (cas de 16 administrations), suivi par le manque de formation en TIC (47\% des répondants), puis le manque de mobilisation autour du projet TIC avec $40 \%$ des répondants et enfin le sentiment d'isolement avec $7 \%$ des répondants comme le montre le graphique 7 ci-après : 
GRAPHIQUE 7 : Fréquence des AP par cause de résistance au changement

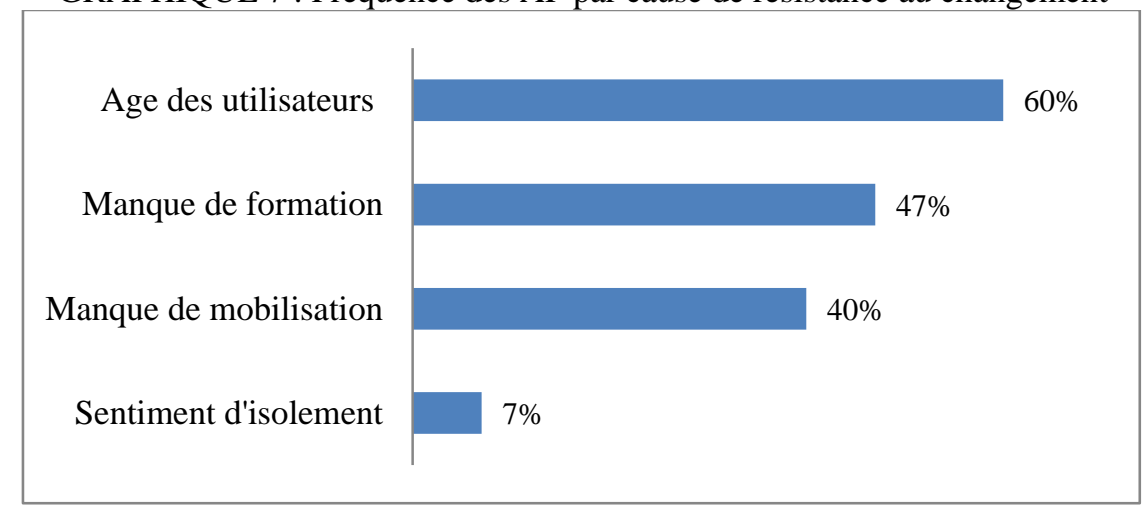

\section{Difficultés de mise en ouvre des TIC}

12 administrations (40\%) ont déclaré que la sécurité et la confidentialité de l'information restent le principal problème rencontré lors de l'introduction des TIC, suivi par la fréquence des coupures de connexion et le désordre informationnel avec un taux de $27 \%$ respectivement (pour 8 entités). De plus, l'utilisation de l'Internet à des fins personnelles est également préoccupante. Dans $77 \%$ des cas (23 administrations), le personnel l'utilise pour la lecture de la presse électronique; $17 \%$ des administrations (5 entités) ont déclaré que leur personnel l'utilise pour accéder aux réseaux sociaux (Facebook et Twitter notamment), 30\% (9 entités) pour regarder des vidéos et enfin 27\% (8 entités) pour les jeux. Ainsi, l'usage des TIC dans l'administration publique se heurte à de nombreux obstacles comme résumé dans le graphique 8 ci-dessous :

GRAPHIQUE 8 : Classement des difficultés de mise en œuvre des TIC par nature et fréquence d'usage

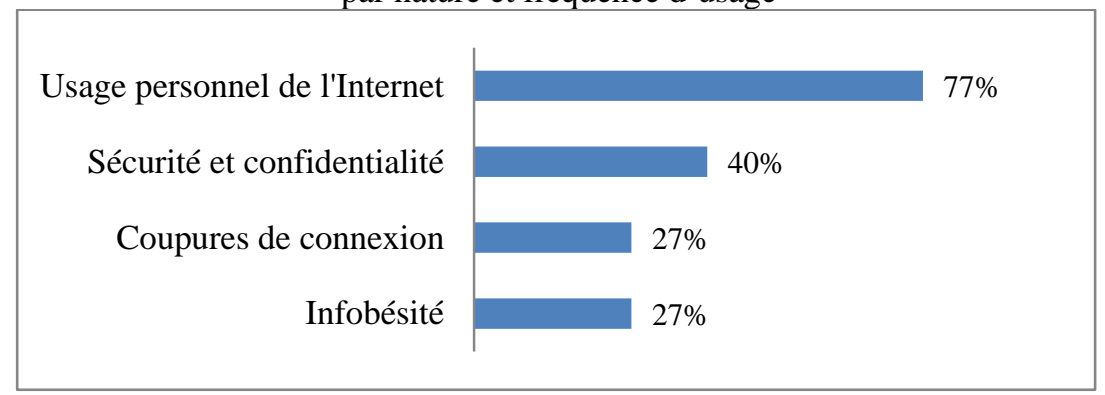

\section{Discussions}

L'Administration marocaine veut faire des TIC un outil de modernisation permettant d'accompagner la réforme administrative au Maroc entamée depuis les années 1990. L'analyse des résultats nous permet de proposer des pistes de réflexion pour relever ce défi et enrichir la compréhension de l'objet de notre étude : 
- la majorité des administrations évolue vers une administration électronique à l'interne comme à l'externe à travers la mise en place de sites Web, de télé-services, l'e-RH, etc. Toutefois, les services interactifs de type Groupeware et Workflow se font rares et le niveau d'utilisation des services en ligne par le personnel, les entreprises et les citoyens reste encore faible.

- l' l'introduction des TIC dans l'Administration publique a entraîné une réorganisation du travail : nouvelle division, simplification/automatisation des tâches administratives et meilleure coordination. Toutefois, les TIC n’ont pas contribué à la réduction des niveaux hiérarchiques et les activités de gestion de connaissances, l'e-Learning, l'évaluation et l'établissement et le suivi des tableaux de bord sociaux sont moins informatisées. Or ce sont ces activités qui génèrent, aujourd'hui, le plus de valeur ajoutée en termes de développement des compétences.

- $\quad$ la FRH devient plus flexible, extravertie et communicante ce qui peut faire d'elle une référence pour les autres fonctions de l'organisation. Elle gagne en efficacité et efficience car les TIC ont permis le travail en groupe, un gain de temps, des économies de ressources, l'amélioration de la qualité du travail, le raccourcissement des délais de traitement des dossiers. Toutefois, elles ne peuvent se substituer totalement au papier, au téléphone et fax et sont encore perçues comme des outils de communication complémentaires.

- les DRH constatent des dysfonctionnements organisationnels notables qui peuvent pénaliser l'efficacité de la FRH en lien avec l'utilisation des TIC. La sécurité des données du système constitue leur principale préoccupation, suivie par la fréquence de coupures de connexion puis de la surcharge informationnelle. De plus, la majorité des employés utilisent l'Internet pour des besoins hors travail. La circulation et le partage d'information, la transparence, l'ouverture se développent grâce aux technologies de l'information uniquement dans les services qui ont déjà ces caractéristiques. Ces dysfonctionnements interpellent le leadership des cadres pour insuffler une culture TIC basée sur le sens éthique et la mobilisation de toutes les parties prenantes pour une pleine réussite du projet d'informatisation de l'Administration.

- l'avènement des TIC a entrainé une modification des conditions du travail. Le rythme du travail s’intensifie et la complexité de certaines tâches augmente ce qui constitue une source de stress potentielle pour le personnel. Toutefois, dans la majorité des cas, cette situation n’a pas altéré la santé mentale des utilisateurs par la perception d'un sentiment d'isolement et n'a pas non plus alimenté l'apparition de conflits au travail. Les TIC ont créé de nouveaux modes de coopération et coordination capables de renforcer les liens sociaux au sein de l'administration. A l'inverse des entreprises privées 
où l'utilisation massive des TIC s'est traduite dans de nombreux cas par une vraie détresse psychologique.

- $\quad$ les TIC favorisent le renforcement du contrôle des utilisateurs dans le cadre d'un management par objectifs axé sur les résultats. Le personnel des administrations dispose de plus d'autonomie dans l'exécution du travail. Toutefois cette autonomie reste limitée à certaines activités basiques qui ne nécessitent pas une intervention de la hiérarchie et ne s’accompagne pas d’une véritable décentralisation du pouvoir.

- $\quad$ les TIC ont contribué au développement du niveau de compétence des utilisateurs, de leur implication et motivation, de leur capacité d'anticipation et ont encouragé l'esprit d'initiative. Toutefois, les TIC ne profitent pas à l'ensemble du personnel et donc leur impact sur les savoir et savoir-faire organisationnels reste limité.

- certains responsables RH n’ont pas enregistré des résistances à la suite de l'introduction des TIC, d'autres constatent peu de résistances au changement. Celles-ci se manifestent soit par le refus de l'utilisation des TIC, soit par le refus de s'occuper de nouveaux dossiers. L’âge de l'utilisateur est cité comme cause principale de résistance, suivi par le manque de formation dans le domaine des TIC et enfin le manque de mobilisation autour du projet d'intégration des TIC dans l'Administration. Toutefois, ces résistances ne sont pas significatives dans la mesure où elles n’ont pas produit des perturbations au niveau de l'exécution du travail.

- les responsables ressources humaines déclarent un manque de formation dans le domaine des TIC en raison de la faiblesse des budgets alloués. L’investissement en capital humain permettant d'accompagner les projets d'informatisation reste très limité. C'est donc une formation de type apprentissage par la pratique qui domine. De plus, les formations pour les techniques réseaux (Intranet, ERP, Workflow, Groupware, etc.) sont quasiabsentes. L'insuffisance de la formation peut constituer un véritable handicap et réduire les résultats attendus de ces technologies.

\section{Conclusion}

Cette recherche contribue à la compréhension de l'impact des TIC sur la gestion publique au Maroc en particulier dans le domaine de la GRH. Nous avons tenté d’appréhender les différents changements, sous différentes dimensions susceptibles d'être induits par ces technologies. En relation avec les perceptions des responsables $\mathrm{RH}$ ou de gestion du personnel, une attention particulière a été accordée à l'employé en tant qu'utilisateur final et facteur déterminant de la réussite de tout projet d’informatisation. Toutefois, notre investigation s'est basée sur un échantillon restreint qui ne respecte pas la rigueur académique requise pour de telles études mais qui permet néanmoins par sa taille (30 administrations publiques) de conceptualiser une 
idée et certaines tendances actuelles dans le champ d’étude qui nous préoccupe.

Ainsi, dans l'administration publique, l'influence structurante des TIC est synonyme d'un renouvellement des pratiques managériales particulièrement en gestion des ressources humaines. L’accès et le partage de l'information (considérée comme variable stratégique) se démocratisent, des services interactifs au service du personnel voient le jour, la FRH tend à se décentraliser et s'éclater dans le sens d'un meilleur partage des missions en interne pour s'occuper davantage de missions stratégiques. L’interaction entre les différentes fonctions de l'organisation devient un enjeu majeur d'autant plus que le développement récent d'applications directement dédiées à la GRH et l'avènement de l'ERM (Gestion de la relation employé) offre une multitude de choix possible en termes de mise en œuvre et de potentialités pour les services de GRH. Plus spécifiquement, de nombreux DRH voient dans les TIC un moyen innovant permettant de répondre aux carences actuelles des SIRH et au besoin d'un meilleur pilotage de l'activité RH.

Toutefois, une certaine culture informatique est indispensable surtout dans les administrations publiques où les risques d'exclusion sont réels, entre ceux qui savent utiliser un ordinateur et ceux qui ne le savent pas. Ces organisations sont vivement appelées à modifier leurs structures et leurs modes de communication pour arriver à un résultat de création de valeur selon des modèles de gestion transversaux orientés vers les clients internes (les salariés) et externes (usagers). Cette transversalité permettrait plus de flexibilité et d'autonomie nécessaire à une gestion différente des connaissances et à une responsabilisation accrue de tous les collaborateurs. Dans l'administration publique moderne, l'usage des TIC doit impulser une dynamique positive permettant une réduction des paliers hiérarchiques, une décentralisation au juste niveau des responsabilités, des attributions et définitions de fonctions clairement disposées; garants d'une meilleure performance, du moins sociale.

\section{References:}

1. Alecian, S. et Foucher, D. (2002). Le management dans le service public, Paris, France, édition d’Organisation, p.446.

2. Allègre, C.B, \& Andréassian, A.E. (2008). Gestion des ressources humaines, valeur de l'immatériel. De boeck., p. 74.

3. Bailly, F.A., Bourgois, D., Gruère, J.P., Raulet-Croset, N., \& RolandLévy C. (2009). Comportements humains \& Management, 3 ème édition, Pearson Education, p.283. 
4. Beyer, J., Ashmos, D.P. \& Osborn R.N. (1997). Contrasts in Enacting TQM: mechanistic Vs organic ideology and implementation, Journal of Quality Management, vol.1, p.13-29.

5. Boston, J. (2010). Basic NPM ideas and their development, in Tom Christensen and Per Lægreid (dir.), The Ashgate Research Companion to New Public Management, Ashgate Pub.

6. Brilman, J. \& Hérard, J. (2006). Les meilleures pratiques du management, Editions d'Organisation., p.539.

7. Cornet, A. (1995). Temporalité des processus de changements et gestion des ressources humaines, Actes du 6ème Congrès de l'AGRH, Poitiers, 23-24 novembre, p.631-639.

8. DeSanctis, G. (1995). Information technology, in Nigel Nicholson (sous la dir.de), Blackwell Encyclopedic Dictionary of organizational Behavior, Cambridge (Massachussets), Blackwell, p.232-233.

9. Dukan, L. (2003). NTIC et psychologie du changement, Bibliotheque.univers-lille1.fr/grisemine, p.19.

10. Duvernay, F. \& Marchal E. (1994). Règles en action : entre une organisation et ses usages, Revue française de Sociologie, $\mathrm{n}^{\circ} 35$.

11. Godelier, E. (1998). Le changement dans les entreprises : crise ou mutation?, Revue Française de Gestion, n²120, Septembre-Octobre, p.24-36.

12. Gollac, M. (2003), Les métiers face aux technologies de l'information, [http://www.ladocumentationfrancaise.fr/var/storage/rapportspublics/034000182.pdf] consulté le 30/11/2016.

13. Gollac, M., Greenan, N. \& Hamon-Cholet, S. (2000). L'informatisation de l'ancienne économie : nouvelles machines, nouvelles organisations et nouveaux travailleurs, Économie et Statistiques, $\mathrm{n}^{\circ}$ 339-340.

14. Guérin, G. \& Sire B. (1999). L’avenir de la fonction ressources humaines. Aujourd'hui le partenariat d'affaires, demain le reengineering et la revalorisation du rôle de champion des employés, Note du LIRHE, n²84, Janvier.

15. Guérin, G. \& Wils, T. (2002). La gestion stratégique des ressources humaines, Gestion, 27(2), pp. 14-23.

16. Kalika, M. (2002). E-GRH, évolution ou révolution ?, Editions Liaisons.

17. Kalika, M., Guilloux, V., Laval, F. \& Matmati M. (2005). E-RH, réalités managériales, Vuibert.

18. Lachmann, H., Larose, C. \& Pénicaud M. (2010). Bien-être et efficacité au travail : 10 propositions pour améliorer la santé 
psychologiquement au travail, Rapport au Premier ministre, Paris, La Documentation française, février, in Klein T. et Ratier D., 2012.

19. Lasfargue, Y. (2000). Techno mordus, techno exclus ? Vivre et travailler à l'ère du numérique, Éditions d'Organisation.

20. Lasfargue, Y. (2003). Halte aux absurdités technologiques, Edition d'Organisation, Paris.

21. Lameul, G. (2000). Former et échanger par les réseaux : ingénierie de formation à distance, Séminaire inter-IUFM, Avril 2000.

22. Lei, D., Hitt M. \& Bettis R.A. (1996). Dynamic capabilities and strategic management, Journal of Management, p.547-567.

23. Matmati, M. (2000). Les NTIC induisent un nouveau modèle de GRH, Management et conjoncture sociale n580, Mai 2000 ; p.2130.

24. Matmati, M. (2002). L'intranet RH, Outil de la performance organisationnelle?, in La E-RH, Evolution ou Révolution, relever le défi de l'intégration des TIC dans la Fonction Ressources Humaines, Edition Liaisons., p.91-122.

25. Markus, M.L., Manville B. \& Agres, C.E. (2002). What makes a virtual organization work?, MIT Sloan Management Review, vol. 42, p.13-27.

26. Megder, E., Cherkaoui C., Sbihi B., \& Mammass D. (2005). Le eGouvernement et la Modernisation du Secteur Public, 3rd International Conference: Sciences of Electronic Technologies of Information and Telecommunications., March 27-31, Tunisia., p.2.

27. Nahon, S. \& Taskin L. (2009). E-GRH : Enjeux et perspectives : informer, collaborer et impliquer. Edition pro, p. 29 et p.36.

28. OCDE (2003). Les TIC et la croissance économique: Panorama des industries, des entreprises et des pays de l'OCDE., p.77.

29. Palvia, P.C., Palvia S.C. \& Roche E.M. (1996). Global Information Technology and Systems Management: Key Issues and Trends, Nashua (New Hampshire), Ivy League.

30. Peretti, J.M. (1995). Les managers, nouveaux DRH. Mise en œuvre de la fonction partagée, Personnel n³59, Avril 1995, p. 26-30.

31. Peretti, J.M. (2001). E-RH et renouvellement des pratiques managériales, Ressources Humaines et management, 2 Mai, pp.1617.

32. Peretti, J.M. (2003). Dictionnaire des ressources humaines, Paris, Vuibert.

33. Pettigrew, A. (1997). Le new public management conduit à un nouveau modèle hybride public-privé, entretien Revue Française de Gestion, n¹15, p. 113-120. 
34. Picq, T. (2000). Les systèmes intranet de gestion des ressources humaines et leurs impacts sur les hommes et les organisations, Personnel, $\mathrm{n}^{\circ}$ 408, Mars-Avril.

35. Ruël, H. (2005). E-HRM Disaster or Destiny, 1st Biennial European Conference on ICT, the Knowledge Society and Changes in Work will be held in The Hague, The Netherlands.

36. Schilling, M.A. (1998). Technological lockout: an integrative model of the economic and strategic factors driving technological success and failure, Academy of management Review, vol.23, $\mathrm{n}^{\circ} 2$, p.267284.

37. Silva, F. (2001). Devenir e-DRH, Edition Liaisons., p 65 et p. 89-90.

38. Ulrich, D. (2001). From e-business to e-RH, Human Resources Planning, Janvier-Février, p.86-98.

39. Veiga, J. \& Dechant, K. (1997). Wired World Woes: www.help, Academy of Management Executive, vol.1, n 3, p. 73-79.

40. Woiceshyn, J. (1997). The role of the management in the adoption of technology: a longitudinal investigation, Technology Studies, vol.4, $\mathrm{n}^{\circ} 1, \mathrm{p} .62-99$. 